\title{
Le premier atelier (workshop) international du grand projet génome humain : le CEPH
}

\section{Jean Dausset}

\section{RÉFÉRENCES}

1. Nathans D, Smith H. Restriction endonucleases in the analysis and restructuring of DNA molecules. Ann Rev Biochem 1975; 44: 273-93.

2. Southern EM. Detection of specific sequences among DNA fragments. $J \mathrm{Mol}$ Biol 1975 ; 98 : 503-17.

3. Kan YW, Dozy AM. Polymorphism of DNA sequence adjacent to human $\beta$-globin structural gene : relationship to sickle mutation. Proc Natl Acad Sci USA 1978; 75 : 5631-5.

4. Botstein D, White RL, Skolnick M, et al. Construction of a genetic linkage map in man using restriction fragment length polymorphisms. Am J Hum Genet $1980 ; 32$ : 314-31.

5. White R. DNA Sequence polymorphisms revitalize linkage approaches in human genetics. Trends Genet $1985 ; 1$ : 177-81.

6. Jeffreys AJ, Wilson V, Thein SL. Hypervariable minisatellite regions in human DNA. Nature $1985: 316: 76-9$.

7. Weber JL, May PE. Abundant class of human DNA polymorphism which can be typed using the polymerase chain reaction. Am J Hum Genet 1989 ; 44 : 388-96.

8. Lit M, Luty JA. A hypervariable microsatellite revealed by in vitro amplification of a dinucleotide repeat within the cardiac muscle actin gene. Am J Hum Genet 1989 ; $44: 397-401$.

\section{ADRESSE}

J. Dausset : professeur honoraire au Collège de France. CEPH, 27, rue Juliette Dodu, 75010

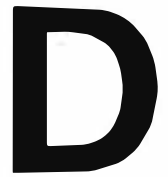

epuis de longues années un travail de patience est mené par les généticiens pour établir la localisation des gènes humains sur la molécule d'ADN. Les réunions bi-annuelles - dont l'avantdernière, la neuvième, s'est tenue à Paris sous la conduite de Jean Frézal (HGM9) - permettent de confronter les résultats des divers chercheurs et de localiser des marqueurs qui étaient pendant longtemps demeurés rares et espacés. La " carte génétique " a ainsi progressé lentement.

La biologie moléculaire a permis d'en accélérer grandement l'établissement. L'histoire débute avec la découverte des enzymes de restriction qui valut, en 1978, le prix Nobel à W. Arber, D. Nathans et K. Smith [1]. Grâce à ces outils, E.M. Southern invente, en 1975, une technique qui porte son nom, par laquelle on décèle aisément la taille des fragments d'ADN sélectionnés par les enzymes de restriction [2]. Sa première application spectaculaire à la médecine fut la démonstration par Y.W. Kan de l'existence d'un polymorphisme portant sur la taille des fragments (restriction fragment length polymorphism ou RFLP) voisin du gène de l'hémoglobine permettant de distinguer les malades ou les porteurs de gènes d'hémoglobine défectueuse [3]. Le diagnostic prénatal de la thalassémie et de l'anémie falciforme était ainsi devenu possible. En 1980, D. Botstein, R. White, R. Davis et M. Skolnick [4] élaborent, à partir de ces prémices, une stratégie capable de permettre l'établissement rapide d'une carte génétique et en particulier celle de l'homme. Ils envisagent de traiter systématiquement le génome humain par diver- ses enzymes et de révéler les fragments ainsi obtenus à l'aide de "sondes ", c'est-à-dire de courtes séquences d'ADN. Certains fragments se révéleront de taille variable selon les individus. Un polymorphisme nouveau, en nombre quasi indéfini, viendra ainsi s'ajouter aux quelques systèmes sanguins tissulaires, enzymatiques ou protéiques jusque-là connus. Ce fut une véritable révolution. $\mathrm{R}$. White, le premier, démontra le bien-fondé de ce concept en le mettant lui-même en œuvre [5].

A Paris, de nombreuses familles bénévoles avaient été rassemblées pour suivre la ségrégation des antigènes HLA. C'est alors qu'avec Daniel Cohen, animé de "l'esprit" des workshops internationales d'histocompatibilité, nous proposons que ce matériel familial serve de point commun à l'ensemble des laboratoires intéressés à la cartographie du génome humain. Celle-ci sera évidemment grandement accélérée si les mêmes familles sont étudiées par de nombreux laboratoires et si les résultats sont rassemblés et analysés centralement. Ray White adhère à ce projet et généreusement offre l'apport de larges familles qu'il avait déjà étudiées. Ainsi est né, en 1983, le Centre d'étude du polymorphisme humain (CEPH), auquel s'associent H. Cann, J.M. Lalouel et M. Lathrop.

Pendant plusieurs années, les RFLP sont la source essentielle des nouveaux marqueurs. Plus récemment, grâce à la découverte de A. Jeffreys, des mini-satellites (courtes séquences d'ADN répétitives [6], mais dont le nombre de répétitions peut varier d'un individu à l'autre) et encore plus récemment à la suite des découvertes de J. Weber et M. Litt, celles des micro-satellites $[7,8]$, le polymor- 
phisme de l'ADN s'est considérablement accru, rendant encore plus nécessaire que jamais une analyse centralisée portant sur le même matériel familial.

A l'heure actuelle, le CEPH compte 64 collaborateurs (États-Unis : 37 ; Canada : 2 ; Europe : 20 ; Afrique du Sud : 2 ; Japon : 2, Australie : 1). Les 61 familles dont l'ADN est distribué comportent 809 individus, avec une moyenne de 8,5 enfants par famille. Pour 44 d'entre elles, on dispose, de plus, des quatre grandsparents. Ces familles constituent un matériel exceptionnel dont les lymphocytes et les lymphocytes immortalisés sont gardés précieusement en azote liquide dans trois villes différentes.

Les collaborateurs reçoivent gracieusement l'ADN de tous les individus. Ils s'engagent en échange à tester avec leurs sondes personnelles l'ensemble des parents et les enfants des familles informatives. Les résultats sont renvoyés au CEPH avant publication, sous un format informatique standard. A partir de ces résultats, le CEPH prépare une base de données génotypiques qui peut être analysée par chacun des collaborateurs, en particulier à l'aide des programmes de liaison génétique multiloci élaboré par M. Lathrop et J.M. Lalouel. Deux marqueurs sont d'autant plus proches qu'ils ségrègent plus souvent ensemble. Ainsi petit à petit se construit la carte génétique. Dès maintenant, plus de 1000 sondes décelant des polymorphismes ont été testées sur les familles du CEPH. Il existe déjà sur tous les chromosomes un repère en moyenne tous les 10 centimorgans (cM). On a calculé qu'il faudrait 6000 marqueurs hautement polymorphes pour établir une carte à $1 \mathrm{cM}$. Les collaborateurs du $\mathrm{CEPH}$ établissent entre eux une carte commune. Celle du chromosome 10 est sous presse, les autres sont en voie d'établissement.

Cependant, le but du CEPH n'est pas d'établir une carte pour ellemême mais pour que celle-ci serve de maille dans laquelle les gènes des maladies génétiques pourront être placés. Déjà des familles du CEPH ont aidé à la localisation des marqueurs voisins du gène de la mucoviscidose (chromosome 7), ce qui a $\mathrm{m} / \mathrm{s} n^{\circ} 3 \mathrm{vol}$. 6, mars 90 permis récemment de l'isoler $(\mathrm{m} / \mathrm{s}$ $n^{\circ} 8$, vol. 5, p. 589) et de le caractériser ; ainsi que des gènes de la polypose colique familiale (bras long du chromosome 5), de la neurofibromatose de von Recklinghausen (chromosome 17), du syndrome de l'X fragile et du déficit immunitaire sévère lié au chromosome $X$. Des marqueurs servent déjà ou serviront au diagnostic prénatal de nombreuses maladies.

Il reste sur les 3000 à 4000 maladies géniques beaucoup d'autres gènes à localiser. A cette fin, le CEPH se propose de distribuer prochainement à titre gratuit, aux médecins généticiens et aux chercheurs, des " trousses" composées de sondes régulièrement espacées sur les 23 chromosomes. Testées sur des familles de malades, on peut espérer, en rassemblant un nombre suffisant de familles informatives, observer la ségrégation commune du phénotype maladie avec un ou plusieurs repères polymorphes. Cette stratégie est certes lourde et lente, mais seule capable, à l'heure actuelle, de localiser les gènes d'une maladie monogénique.

Quant aux maladies polygéniques, elles pourront également bénéficier de la carte génétique pour localiser les différents gènes dont la présence simultanée chez un même individu lui confère une susceptibilité particulière. On sait, par exemple, que la présence des allèles HLA-DR3 et DR4 et plus spécialement d'un allèle de $\mathrm{DQ} \beta$ prédispose grandement au diabète juvénile, mais d'autres gènes interviennent sans doute, disséminés dans le génome. Repérer ces gènes permettrait une véritable médecine prédictive rendant possible une prévention efficace et personnalisée pour le diabète, mais aussi pour bien d'autres affections fréquentes dans nos sociétés comme l'hypertension et peut-être certains cancers. Telle sera la médecine du XXI' siècle.

Le grand projet "Génome humain " a fait couler beaucoup d'encre. Il comporte au moins trois volets: la carte génétique, la carte physique et la séquence avec en corollaire la lecture de l'immense bibliothèque de 3,5 milliards de lettres.

Le CEPH a été, dans ce domaine, un précurseur car fondé avant que le projet Génome humain ne soit envisagé. On peut donc dire qu'il constitue le premier atelier (workshop) international du Grand Projet Génome humain. Il aidera, nous l'espérons, à le réaliser au moins dans l'un de ces volets

\section{Summary}

The first international workshop on the Great Human Genome Project (CEPH)

The Centre d'Étude du Polymorphisme humain $(\mathrm{CEPH})$ is a new project research institute that makes available to the scientific community DNA samples from a panel of large families for the determination of genotypes for various DNA polymorphisms which are used for the construction of the genetic map of the human genome. Already this map has been useful for the localization of gene hereditary of diseases.

\section{TIRÉS A PART}

